\title{
Successful management of chemotherapy induced polyneuropathy
}

\author{
B Mahawar ${ }^{*}$ \\ Consultant Physician, MICU, Pain and Palliative Care, Rajiv Gandhi Cancer Institute and Research \\ Centre, Delhi, India.
}

\begin{abstract}
The management of chemotherapy induced polyneuropathy (CIPN) in a patient with pre-existing, painful diabetic neuropathy $(\mathrm{DN})$ is reported. It was managed successfully with pregabalin and duloxetine.
\end{abstract}

Keywords: Chemotherapy induced polyneuropathy (CIPN); diabetic polyneuropathy; carboplatin; docetaxel

\section{Introduction}

CIPN is complex and can be often severe and resistant to treatment. When superimposed on painful diabetic neuropathy then it can be intractable. It is a known fact that platinum ${ }^{1}$ and taxane based salts are neurotoxic and can affect peripheral nerves. Conservative management with co- analgesics e.g. pregabalin and duloxetine gives good results. ${ }^{2}$

\section{Case report}

58yr old female, with a carcinoma of the right breast, post modified radical mastectomy (MRM) stage PT2N0 (ER+ estrogen receptor positive, PR+ progestrone receptor positive, HER2 neu $3+$ from human epidermal growth factor receptor 2 are the hormonal status of patient) was on adjuvant chemotherapy (CT). She is a known diabetic since $15 y r s$ and was normotensive. Her blood sugar levels were uncontrolled and had been neglected for the past several years, and was poorly compliant on oral hypoglycemic drugs.

For the past 3yrs, she was suffering from gradually increasing pain in bilateral feet below ankle, glove and stocking type distribution; however, no
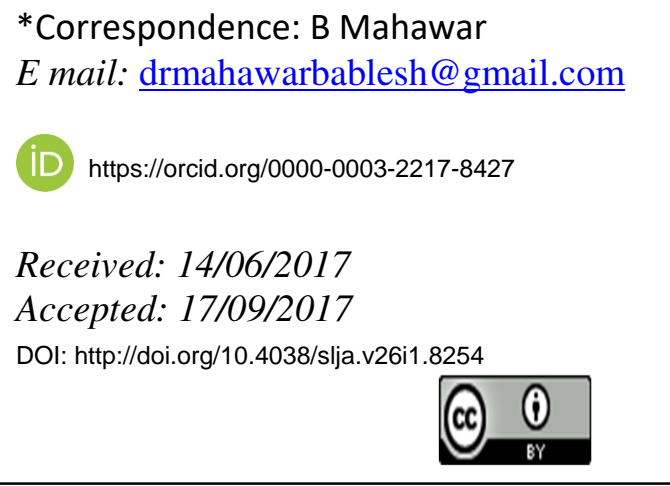

treatment was sought. Post MRM surgery, (Histopathological examination-invasive duct carcinoma) she was planned for targeted chemotherapy (docetaxel 120mg, carboplatin $540 \mathrm{mg}$ and trastuzumab 548mg). Post $10^{\text {th }}$ day of chemotherapy patient complained of severe bilateral lower limb pain and pain relief team was consulted. Pain was stabbing type with burning sensation described in the feet, shin and thighs, heaviness and numbness migrating up to the shin and thighs of both legs accompanied with gait imbalance, making her unable to walk. She characterized pain intensity at 9/10. Pain Detect Tool showed score of $>20$ suggesting neuropathic type involving both small and large diameter fibers. On examination motor power was grade $4 / 5$ for toe dorsiflexion and plantar flexion. Tone was normal, there was sensory deficit and absent knee and ankle reflexes. Plantar response was flexor bilaterally. There was decreased pinprick and temperature perception to the knee bilaterally. The patient had absent vibratory perception in the toes. Proprioception was mildly reduced. Coordination was normal on finger -nose-finger and heel- kneeshin testing bilaterally. EMG (electromyographic) and NCV (nerve conduction velocity) studies were done suggesting severe mixed motor sensory polyneuropathy of bilateral lower limbs. $\mathrm{HbA}_{1 \mathrm{C}}$ was 10.2. Mentally patient was agitated and hopeless, entire family was apprehensive and worried about her pain. After due deliberation, a diagnosis of CIPN superimposed on preexisting diabetic neuropathy ${ }^{3}$ was made. The background pain of diabetic neuropathy was aggravated with the first cycle of chemotherapy. Patient was started on pregabalin $75 \mathrm{mg}$ and duloxetine $20 \mathrm{mg}$ orally one at bed time. Pregabalin was increased to $150 \mathrm{mg}$ and duloxetine was increased to $20 \mathrm{mg}$ TDS over a 
two-week period. Tramadol and paracetamol combination was added to take care of initial period of pain till co- analgesics started acting. Meantime patient was encouraged to strive for better glycaemic control to prevent further complications related to diabetes mellitus. The oncology team was also consulted and they modified their regimen to reduce dosage of docitaxel and carboplatin drugs notorious for inducing CIPN. Patient responded well to the treatment, over two weeks' time with pain score $2 / 10$, better mental outlook; she was more upbeat about her disease and overall her family members were relieved and happy seeing her painless condition and positive attitude.

\section{Discussion}

DN is the most common form of neuropathy worldwide and can involve proximal, distal, somatic and autonomic nerves contributing to substantial morbidity and mortality. The proximal muscle weakness often is associated with reduced or absent extremity reflexes. The most common problem is the diffuse numbness and pain that is due to peripheral neuropathy, which begins in the feet and moves up into the legs. Neuropathy can also affect the hands, but not usually until the late stages. CIPN is a common adverse side effect of many of the most effective drugs and the diabetic patient, already at risk of nerve compression and neuropathy, is more likely to be prone to peripheral nerve damage. Diabetes mellitus has been identified as the most important risk factor for CIPN.

The chemotherapeutic agents at a high risk for causing CIPN are platinum based agents, taxanes, and vinca alkaloids. CIPN is a complex phenomenon. No single pathophysiologic process has yet been identified to explain the various neuropathies that occur after exposure to different chemotherapeutic agents. While it is tempting to consider CIPN as a single entity, it may be more pragmatic and useful to consider each neuropathy separately as a specific side effect of a specific drug class, as successful prevention and treatment may differ. However, they are dose dependent and progressive and change of therapy or reduction in dosage (as was done in our patient) is needed.

Many treatment options have been tried for CIPN such as NSAIDs, opioids, tricyclic antidepressants, gabapentin and topical applications, but there is lack of consensus and no specified treatment regimen. Duloxetine (a neuronal selective serotonin-norepinephrine re-uptake inhibitor) and pregabalin (a gamma amino butyric acid analogue) have been used comparatively recently and show great promise. The successful management of severe CIPN in our patient with these two drugs adds to the evidence regarding their efficacy and safety.

It must be stressed that awareness of the problem and accurate assessment of the severity of the neuropathy and efficacy of treatment are invaluable in ensuring a better quality of life and improves compliance with essential chemotherapy.

\section{References}

1. Chaudhry V, Rowinsky EK, Sartorius SE, et al. Peripheral neuropathy from taxol and cisplatin combination chemotherapy: clinical and electrophysiological studies. AnnNeurol. 1994;35(3):304-11. 10.1002/ana.410350310 [Pub Med] https://doi.org/10.1002/ana.410350310

2. Kautio A, Haanpää M, Kautiainen H, et al. Burden of chemotherapy-induced neuropathy--a crosssectional study. Support Care Cancer. 2011;19(12):1991-6. 10.1007/s00520-010-1043-2 [PubMed] https://doi.org/10.1007/s00520-010-1043-2

3. Bril V, England J, Franklin GM, etal. Evidence based guideline: Treatment of painful diabetic neuropathy: Report of the American Academy of Neurology, the American Association of Neuromuscular and Electrodiagnostic Medicine and the American academy of physical Medicine and Rehabilitation. Neurology 2011; 76;1758-1765. Epub April,2011

https://doi.org/10.1212/WNL.0b013e3182166ebe

PMid:21482920 PMCid:PMC3100130 